\title{
BLOCKS OF SMALL DEFECT
}

\author{
ALBERTO ESPUELAS AND GABRIEL NAVARRO
}

(Communicated by Warren J. Wong)

\begin{abstract}
A group of odd order $G$ with $O_{p}(G)=1$ has a block of defect less than $[n / 2]$, where $p^{n}=|G|_{p}$. In addition, if $G$ is supersolvable by nilpotent, $G$ has a block of defect zero.
\end{abstract}

\section{INTRODUCTION}

It is an interesting problem to give necessary and sufficient conditions for the existence of $p$-blocks of defect zero. If a finite group $G$ has a block of defect zero, it is well known that $O_{p}(G)=1$, although, of course, this is not a sufficient condition. Ito proved [3, X.6.5] that if $G$ is a nilpotent by nilpotent group of odd order, then $G$ has a block of defect zero iff $O_{p}(G)=1$.

Recent results on regular orbit theorems have associated conditions for the existence of blocks of defect zero [1]. For groups of odd order, with $O_{p}(G)=1$, the exceptions are essentially nilpotent by supersolvable as Theorem 1 of [1] shows us.

In general, we try to find the smallest defect $d(B)$ of a block $B$ of $G$. This is given in Theorem A below.

Theorem A. Let $G$ be a (solvable) group of odd order such that $O_{p}(G)=1$ and $|G|_{p}=p^{n}$. Then $G$ contains a p-block $B$ such that $d(B) \leq[n / 2]$. The bound is best possible.

It is not true in general that there exists a block $B$ with $d(B) \leq[n / 2]$, as $G=A_{7} \quad(p=2)$ shows us.

By work of Michler and Willems [7, 8] every simple group except possibly the alternating group has a block of defect zero for $p \geq 5$. Perhaps the following has an affirmative answer.

Question. If $G$ is a finite group with $O_{p}(G)=1, p \geq 5$, and $|G|_{p}=p^{n}$, does $G$ contain a block of defect less than $[n / 2]$ ?

If $\mathscr{F}$ is the class of supersolvable by nilpotent groups, we can prove the following.

Received by the editors June 8, 1990 and, in revised form, September 24, 1990.

1980 Mathematics Subject Classification (1985 Revision). Primary $20 \mathrm{C} 15$.

Key words and phrases. Block, defect, defect zero.

Both authors are partially supported by DGICYT. 
Theorem B. Let $G \in \mathscr{F}$ with $|G|$ odd. Then $G$ has a p-defect zero character if and only if $O_{p}(G)=1$.

\section{BLOCKS OF DEFECT LESS THAN $[n / 2]$}

The next lemma is a key tool for proving Theorem A. We denote by $F(G)$ the Fitting subgroup of the group $G$.

2.1. Lemma. Let $G$ be a group of odd order and let $V$ be a faithful and irreducible $K G$ module, $\operatorname{char}(K)$ being odd. Then there exists a normal subgroup $H$ of $G$ (possibly $H=1$ ) and a vector $v \in V$ such that: $C_{G}(v)^{\#} \subseteq H-F(H)$ and $H / F(H)$ is abelian.

Proof. See the proof of Theorem 3.1 of [2].

Theorem A. Let $G$ be a (solvable) group of odd order such that $O_{p}(G)=1$ and $|G|_{p}=p^{n}$. Then $G$ contains a p-block $B$ such that $d(B) \leq[n / 2]$. The bound is best possible.

Proof. Induction on $|G|$. Consider $\bar{G}=G / \Phi(G)$. As $F(G / \Phi(G))=F(G) /$ $\Phi(G)$, we have that $O_{p}(\bar{G})=1$ and $|G|_{p}=|\bar{G}|_{p}$. If $\Phi(G) \neq 1$, then the result is true for $\bar{G}$. Let $\bar{B}$ be a $p$-block of $\bar{G}$ such that $d(\bar{B}) \leq[n / 2]$. By Lemma V.4.3. of [3], there exists a $p$-block $B$ of $G$ such that $d(B)=d(\bar{B})$. Hence we may àssume that $\Phi(G)=1$.

Now, $V=\operatorname{Irr}(F(G))$ is a faithful and completely reducible $G / F(G)$-module (over different fields, possibly).

Put $V=V_{1} \oplus \cdots \oplus V_{t}$, where each $V_{i}$ is an irreducible $G$-module.

Define $K_{i}=C_{G}\left(V_{i}\right), \bar{G}_{i}=G / K_{i}$ and use the bar convention. By the lemma above, there exists a normal subgroup $H_{i}$ of $G$ containing $K_{i}$ and an element $\lambda_{i} \in V_{i}$ such that $C_{\bar{G}_{i}}\left(\lambda_{i}\right)^{\#} \subseteq \bar{H}_{i}-F\left(\bar{H}_{i}\right)$. Furthermore, $\bar{H}_{i} / F\left(\bar{H}_{i}\right)$ is abelian.

Consider $\lambda=\lambda_{1} \times \cdots \times \lambda_{t}$ and put $C=C_{G}(\lambda)$.

We may view $G / F(G)$ as a subgroup of $\bar{G}_{1} \times \cdots \times \bar{G}_{t}$. This shows that $C \subseteq$ $F_{3}(G)$ and $C \cap F_{2}(G)=F(G)$, where, as usual, $F_{1}(G)=F(G), F_{i}(G) / F_{i-1}(G)$ $=F\left(G / F_{i-1}(G)\right)$.

We consider separately two cases.

(1) $|C|_{p} \leq|G|_{p}^{1 / 2}$.

Take $\chi \in \operatorname{Irr}(G)$ lying over $\lambda$ and let $B$ be the $p$-block of $G$ containing $\chi$. As $F(G)$ is a $p^{\prime}$-group, Lemma V.2.3 of [3] shows that every irreducible character $\psi$ in $B$ has $\lambda$ as an irreducible constituent. Now $\psi(1)_{p} \geq$ $\left|G: I_{G}(\lambda)\right|_{p} \geq|G|_{p}^{1 / 2}$.

The result follows.

(2) $|C|_{p}>|G|_{p}^{1 / 2}$.

Now $\left|F_{3}(G) / F_{2}(G)\right|_{p}>|G|_{p}{ }^{1 / 2}$.

Let $P / F_{2}(G)$ be a Sylow $p$-subgroup of $F_{3}(G) / F_{2}(G)$. Where $Y=$ $O_{p^{\prime}}(F(G / F(G))$, observe that $W=\operatorname{Irr}(Y / \Phi(Y))$ is a faithful and completely reducible $P / F_{2}(G)$-module.

By Gow's regular orbit theorem $[4,2.6]$, we have $\mu \in W$ such that $C_{P}(\mu)=$ $F_{2}(G)$. We may view $\mu$ as a character of the preimage $X$ of $Y$ in $G$. Observe that $X$ is a $p^{\prime}$-group. Take $\chi \in \operatorname{Irr}(G)$ lying over $\mu$. Now $\chi$ lies over an irreducible character $\psi$ of $P$ lying over $\mu$. Clearly, $\psi(1)_{p} \geq\left|F_{3}(G) / F_{2}(G)\right|_{p}>$ 
$|G|_{p}{ }^{1 / 2}$. As $P$ is normal in $G$, we have $\chi(1)_{p} \geq \psi(1)_{p}$. Now the same argument as in case (1) completes the proof.

To show that the bound is best possible, consider two odd primes $p$ and $q$ such that $q \equiv 1(\bmod p)$. Now $\left(q^{p}-1\right) / q-1$ is odd and $\left.\left(q^{p}-1\right) / q-1\right)_{p}=p$. Now $C_{p} \ltimes G F\left(q^{p}\right)^{*}$ acts on $G F\left(q^{p}\right)^{+}, C_{p}$ acting as a Galois automorphism. Thus $H=C_{p} \ltimes C_{\left(q^{p}-1 / g-1\right)}$ acts on $V=C_{q} \times \cdots \times C_{q}$ ( $p$ times) and $V$ does not contain any regular $H$-orbit. Consider $X=H \ltimes V$ and let $G$ be the direct product of $m$ copies of $X$. Then, if $\chi \in \operatorname{Irr}(G)$, we have $\chi(1)_{p} \leq p^{m}$. Thus $d(B) \geq m$ for any $p$-block of $G$ and $|G|_{p}=p^{2 m}$.

\section{SUPERSOLVABLE BY NILPOTENT GROUPS}

We need a regular orbit theorem for proving Theorem B. It is a slight generalization of $[4,2.6]$

3.1. Theorem. Let $G$ be a group of odd order and let $V$ be a faithful irreducible $K G$-module, where $K$ is a field of odd characteristic $q$. Assume that $G$ is $p$ nilpotent for some prime $p \neq q$ and that

$$
V_{{p^{\prime}}^{\prime}(G)}=V_{1} \oplus \cdots \oplus V_{t},
$$

where each $V_{i}$ is a 1-dimensional $K O_{p^{\prime}}(G)$-module. Then there exists $v \in V$ such that $C_{G}(v)=1$.

Proof. We induct on $|G|$.

Write $H=O_{p^{\prime}}(G)$. We claim that $V_{H}$ is not homogeneous. Let $W$ be an irreducible submodule of $V_{H}$ and let $I=\{g \in G \mid W g \cong W$ as $K H$-modules $\}$. By hypothesis, $\operatorname{dim}_{K} W=1$.

If $I=G$, then $W$ is a faithful 1-dimensional $K H$-module and $V$ is $K H$ homogeneous.

A well-known argument implies that $H$ is contained in $Z(G)$. Then $G$ is nilpotent and since $V$ is faithful, $q$ does not divide $|G|$. Now, Gow's regular orbit theorem [4. 2.6] gives us $v \in V$ such that $C_{G}(v)=1$.

Thus, we may assume that $V_{H}$ is not homogeneous. Then, we may find a normal subgroup $N$ of $G$ of index $p$ and an irreducible $K N$-module $U$ with $U^{G}=V$ such that $W$ is an irreducible submodule of $U_{H}$. By Clifford's theorem, we may apply induction to $N$ and find $u \in U$ such that

$$
C_{N}(u)=C_{N}(U) .
$$

If $x_{1}, \ldots, x_{p}$ is a set of coset representatives of $N$ in $G$, by using the facts that $n u \neq-u \quad \forall n \in N$ (because $q$ and $|G|$ are odd) and that core ${ }_{G}\left(C_{N}(u)\right)=$ $C_{G}(V)=1$, it can be checked that $C_{G}(v)=1$, where

$$
v=u \otimes x_{1}+\cdots+u \otimes x_{p-1}-u \otimes x_{p} .
$$

This finishes the proof of the theorem.

Recall that a group $G$ has a $p$-block of defect zero if it has an irreducible character $\chi$ with $\chi(1)_{p}=|G|_{p}$.

3.2. Lemma. (a) Let $H$ be a subgroup of $G$ and let $\mu \in \operatorname{Irr}(H)$, with $\mu^{G}=$ $\chi \in \operatorname{Irr}(G)$. Then $\chi(1)_{p}=|G|_{p}$ if and only if $\mu(1)_{p}=|H|_{p}$.

(b) Let $N$ be a normal subgroup of $G$, let $\theta \in \operatorname{Irr}(N)$ and let $\chi \in \operatorname{Irr}(G \mid \theta)$. 
If $\chi(1)_{p}=|G|_{p}$, then $\theta(1)_{p}=|N|_{p}$. Conversely, if $\theta(1)_{p}=|N|_{p}$, and $|G: N|$ is a $p^{\prime}$-number, then $\chi(1)_{p}=|G|_{p}$.

Proof. (a) Since $|G: H| \mu(1)=\chi(1)$, we have that

$$
|G|_{p} / \chi(1)_{p}=|H|_{p} / \mu(1)_{p} .
$$

(b) By (a) and the Clifford correspondence, we may assume that $\chi_{N}=u \theta$, for some integer $u$. Thus $|G|_{p}=u_{p} \theta(1)_{p}$.

We know that $\theta(1)_{p}$ divides $|N|_{p}$, and that $u_{p}$ divides $|G / N|_{p}[6,11.29]$. Since $|G / N|_{p}|N|_{p}=|G|_{p}$, necessarily, we will have

$$
u_{p}=|G / N|_{p} \text { and } \theta(1)_{p}=|N|_{p} .
$$

Conversely, suppose that $\theta(1)_{p}=|N|_{p}$, and that $|G: N|$ is a $p^{\prime}$-number. Let $T=I_{G}(\theta)$, let $\mu \in \operatorname{Irr}(T)$ the Clifford correspondent of $\chi$ over $\theta$, and write $\mu_{N}=v \theta$, for some integer $v$. Then $v$ is a $p^{\prime}$-number,

$$
\mu(1)_{p}=\theta(1)_{p}=|N|_{p}=|T|_{p},
$$

and by (a) the proof is complete. Now we can prove Theorem B.

Theorem B. Let $G \in \mathscr{F}$ with $|G|$ odd. Then $G$ has a p-defect zero character if and only if $O_{p}(G)=1$.

Proof. If $\chi \in \operatorname{Irr}(G)$ with $\chi(1)_{p}=|G|_{p}$, let $\theta$ be an irreducible constituent of $\chi_{O_{p}(G)}$. By part (b) of the lemma above, $\theta(1)=O_{p}(G)$, and thus $O_{p}(G)=1$.

Suppose that $G \in \mathscr{F}$ with $|G|$ odd. We prove that $G$ has a $p$-defect zero character by induction on $|G|$.

Let $N$ be a normal supersolvable subgroup of $G$ such that $G / N$ is nilpotent. Let $P$ be a Sylow $p$-subgroup of $G$ and let $F=F(G)$ be the Fitting subgroup of $G$.

First we prove that $G / N$ is a $p$-group. Since $G / N$ is nilpotent, $P N$ is a normal subgroup of $G$. Since $O_{p}(P N)=1$, by induction and the lemma, we may assume that $P N=G$.

We claim that $G$ is $p$-nilpotent with $O_{p^{\prime}}(G)$ supersolvable. Let $H$ be a Hall $p^{\prime}$-subgroup of $G$. Since $G / N$ is a $p$-group, $H$ is contained in $N$. Since $N$ is supersolvable, $N / F(N)$ is abelian. But $F(N)$ is a $p^{\prime}$-group, because $O_{p}(F(N))=1$. Thus $F(N) \subseteq H \subseteq N$.

This implies that $H$ is normal in $G$, as wanted.

Now, since $F / \Phi(G)=F(G / \Phi(G)), O_{p}(G / \Phi(G))=1$, and we may assume that $\Phi(G)=1$.

Write $F=E_{1} \times \cdots \times E_{s}$, where the $E_{i}$ 's are minimal normal subgroups of $G$.

If $E$ is any normal subgroup of $G$ contained in $F$, we claim that there exists $\lambda \in \operatorname{Irr}(E)$ such that $I_{G}(\lambda)=C=C_{G}(E)$.

Suppose that $|E|$ is a $q$-power, for a prime $q$. Observe that $E \subseteq H$. Since $H$ is supersolvable, by Clifford's theorem $E_{H}$ is a direct sum of 1-dimensional $K E$-submodules, and so it is $E_{H C / C}, K=G F(q)$.

Let $\hat{E}=\operatorname{Irr}(E)$. Then $\hat{E}$ is a faithful irreducible $K[G / C]$-module.

Since $E_{H C / C}=X_{1} \oplus \cdots \oplus X_{t}$, where the $X_{i}$ 's are 1-dimensional $K[H C / C]$ submodules, it follows that

$$
\hat{E}_{H C / C}=\widehat{X_{1}} \oplus \cdots \oplus \widehat{X}_{t},
$$


where $\widehat{X}_{i}=\operatorname{Irr}\left(X_{i}\right)$ is a 1-dimensional irreducible $K[H C / C]$-module. By Theorem 3.1 above, the claim is proved.

Now, let $\lambda_{i} \in \operatorname{Irr}\left(E_{i}\right)$ such that $I_{G}\left(\lambda_{i}\right)=C_{G}\left(E_{i}\right)$, and let

$$
\lambda=\lambda_{1} \times \cdots \times \lambda_{t} .
$$

Then

$$
I_{G}(\lambda)=\bigcap_{i=1, \ldots, s} C_{G}\left(E_{i}\right)=C_{G}(F)=F .
$$

Thus $\lambda^{G} \in \operatorname{Irr}(G), \lambda^{G}$ has $p$-defect zero and the proof is complete.

\section{REFERENCES}

1. A. Espuelas, On the Fitting length conjecture, Arch. Math. 53 (1989), 524-527

2. __ Large character degrees of groups of odd order, Illinois J. Math. (to appear).

3. W. Feit, The representation theory of finite groups, North-Holland, Amsterdam, New York, and Oxford, 1982.

4. R. Gow, On the number of characters in a p-block of a p-solvable group, J. Algebra 65 (1980), 421-226.

5. B. Huppert, Endliche Gruppen, Springer-Verlag, Berlin and New York, 1967.

6. I. M. Isaacs, Character theory of finite groups, Academic Press, New York, 1976.

7. G. Michler, A finite simple group of Lie type has $p$-blocks with different defects, $p \neq 2, \mathrm{~J}$. Algebra 104 (1986), 220-230.

8. W. Willems, Blocks of defect zero in finite simple groups of Lie type, J. Algebra 113 (1988), 511-522.

Departamento de Algebra, Facultad de Ciencias, Universidad de Zaragoza, Zaragoza, SPAIN

Departamento de Algebra, Facultad de Matemáticas, Universitat de Valencia, BurJassot, Valencia, Spain 